\title{
Improving Outcomes in Patients With Sepsis
}

Scott B. Armen, MD, FACS, FCCP, FCCM ${ }^{1}$, Carol V. Freer, MD, FACP ${ }^{1}$, John W. Showalter, MD, MSIS ${ }^{1}$, Tonya Crook, MD, MS, DTM\&H ${ }^{1}$, Cynthia J. Whitener, MD ${ }^{1}$, Cheri West, MS, RN ${ }^{1}$, Thomas E. Terndrup, MD ${ }^{1}$, Marissa Grifasi, PharmD, BCPS ${ }^{1}$, Christopher J. DeFlitch, MD ${ }^{1}$, and Christopher S. Hollenbeak, PhD ${ }^{1}$

${ }^{1}$ The Pennsylvania State University, Hershey, PA

\begin{abstract}
Sepsis mortality may be improved by early recognition and appropriate treatment based on evidence-based guidelines. An intervention was developed that focused on earlier identification of sepsis, early antimicrobial administration, and an educational program that was disseminated throughout all hospital units and services. There were 1331 patients with sepsis during the intervention period and 1401 patients with sepsis during the control period. After controlling for expected mortality, patients in the intervention period had $30 \%$ lower odds of dying (odds ratio $=$ $0.70,95 \%$ confidence interval $[\mathrm{CI}]=0.57$ to 0.84 ). They also had 1.07 fewer days on average in the intensive care unit $(95 \% \mathrm{CI}=-1.98$ to -0.16$), 2.15$ fewer hospital days $(95 \% \mathrm{CI}=-3.45$ to -0.86), and incurred on average $\$ 1949$ less in hospital costs, although the effect on costs was not statistically significant. Continued incremental improvement and sustainment is anticipated through organizational oversight, continued education, and initiation of an automated electronic sepsis alert function.
\end{abstract}

\section{Keywords}

sepsis; severe sepsis; quality improvement; evidence-based medicine; interdisciplinary health team; outcomes assessment

Contemporary reports estimate approximately 751000 sepsis cases annually in the United States, resulting in 215000 deaths and nearly $\$ 17$ billion in annual total costs. ${ }^{1}$ Because of the anticipated population growth, higher incidence of sepsis in older patients, and the disproportionate growth of the elderly in the US population, 1110000 sepsis cases are projected annually by the year 2020. ${ }^{1}$ Several hospital-based studies report varying hospital and intensive care unit (ICU) occurrence rates, ranging from $2 \%$ to $11 \%$ of all hospital and ICU admissions. ${ }^{1}$ In the noncardiac ICU, sepsis has been determined to be the most common cause of death. ${ }^{2}$ In 1995 alone, sepsis resulted in $9.3 \%$ of all deaths in the United States and equaled the number of deaths caused by acute myocardial infarction. ${ }^{1}$ Over the

Reprints and permissions: sagepub.com/journalsPermissions.nav

Corresponding Author: Christopher S. Hollenbeak, PhD, The Pennsylvania State University, College of Medicine, 500 University Drive, H151, Hershey, PA 17033-0850. chollenbeak@psu.edu.

Declaration of Conflicting Interests

The authors declared no potential conflicts of interest with respect to the research, authorship, and/or publication of this article. 
last several decades, hospital mortality from sepsis has ranged from $25 \%$ to $80 \%$ in the majority of studies, with a recent meta-analysis of 131 studies reporting a mortality rate of $49.7 \% .^{1,3}$ Despite medical advances such as the availability of potent antibiotics and refined supportive care, the mortality of septic patients remains high. ${ }^{4}$ With an increasing incidence, high mortality, and staggering annual cost, sepsis will place a significant burden on the US health care system in the coming years.

Although the incidence of sepsis and the number of sepsis-related deaths are increasing, the overall mortality rate among patients with sepsis does appear to be declining. ${ }^{5}$ This is largely because of the widespread implication of quality initiatives based on the concept of early goal-directed therapy (EGDT) as presented by Rivers et al in 2001.6 EGDT is a comprehensive strategy for treating septic patients that includes the identification of highrisk patients based on early pathogenesis, mobilization of resources for intervention, performance of a consensus-developed protocol to reverse early hemodynamic abnormalities, appraisal of quality indicators to assess compliance, and assessment of outcomes. ${ }^{7}$ EGDT has demonstrated significant outcome benefits in patients with severe sepsis and septic shock, with the original study demonstrating decreased in-hospital mortality from $46.5 \%$ in the standard therapy group to $30.5 \%$ in the EGDT group. ${ }^{6}$ The principles of EGDT were embraced by the Surviving Sepsis Campaign (SSC), which drafted guidelines for management of severe sepsis and septic shock. ${ }^{8,9}$ Subsequently, a performance improvement initiative was developed with the goal of changing clinical behaviors through the implementation of bundles based on the SSC guideline recommendations on process improvement and patient outcomes. This process was implemented in the ICU, emergency department (ED), and wards at 165 sites in the United States, Europe, and South America. As a result, unadjusted hospital mortality decreased from $37 \%$ to $30.8 \%$ over 2 years. Most important, SSC was associated with sustained, continuous quality improvement in sepsis care. ${ }^{10}$

In 1991, the American College of Chest Physicians (ACCP) and the Society of Critical Care Medicine (SCCM) convened a conference in an attempt to standardize the definitions of sepsis. The resultant definitions included the systemic inflammatory response system, sepsis, severe sepsis, and septic shock. ${ }^{2,11}$ Although these definitions have been widely accepted and extensive efforts aimed at early recognition have been undertaken, the diagnosis of sepsis remains challenging. Many other disease states possess similar clinical presentations and may share the same pathophysiological processes. ${ }^{11}$ In fact, an international sepsis survey demonstrated that $86 \%$ (905 of 1058) of physicians agreed that the symptoms of sepsis can easily be misattributed to other conditions. When defining sepsis, only $22 \%$ (114 of 529) of intensivists and 5\% (26 of 529) of other physicians gave the ACCP/SCCM consensus definitions. ${ }^{12}$ Furthermore, in 2008, the updated SSC international guidelines referred to the 1991 consensus definitions but more broadly defined sepsis as infection plus systemic manifestations of infection. ${ }^{9}$ Thus, considerable opportunity exists to improve the early recognition of sepsis by all clinicians, which in turn may greatly facilitate evidencebased treatment and thereby improve outcomes.

Concern over the rate of sepsis and an interest in reducing mortality related to sepsis at the study 491-bed academic teaching institution led to a multidisciplinary initiative to improve 
sepsis outcomes. The initiative was multifaceted and included mandatory education, protocol and systems development, creation of evidence-based treatment algorithms and electronic order sets, and continuous compliance monitoring through the development of electronic quality dashboards. The purpose of this study was to determine whether this initiative significantly improved sepsis outcomes.

\section{Methods}

\section{Initiative}

The research team used EGDT as a guide in developing and implementing a sepsis initiative. The initiative included (a) a baseline survey of clinical staff on knowledge related to sepsis syndromes, (b) development of a sepsis treatment bundle of evidence-based practice, (c) a compulsory education program around sepsis and sepsis care providing continuing medical education credit, and (d) monthly result reporting of sepsis outcomes on the hospital electronic quality dashboard. A customized sepsis bundle based on the SSC guidelines and an initial anchor antibiotic algorithm were developed incorporating the Penn State Hershey Medical Center (PSHMC) patient population, care pathways, and pharmacy formulary from July through September 2010. The focus of the bundle was early recognition and assessment of sepsis and rapid anchor antibiotic administration and initial fluid resuscitation. The next steps were prompt identification of suspected infection source and assessment for end-organ dysfunction, followed by antimicrobial administration based on the presumed source of infection. The research team also designed and implemented a severe sepsis/septic shock bundle. Sepsis and severe sepsis/septic shock bundle electronic order sets for the ED and inpatient units were developed and approved. All patients with suspected sepsis were included. A majority of these patients presented initially to the ED and were admitted from the ED with a diagnosis of sepsis. Others arrived with suspected sepsis as direct admissions from referring centers, while a minority developed manifestations of sepsis after admission to the PSHMC for different diagnoses.

The educational program began on October 1, 2010. Sepsis bundle order sets were implemented December 1, 2010, in the ED and ICUs and then hospital wide on January 1, 2011. Thus, the premeasurement reference period was from January 1, 2009, through September 30, 2010. The postimplementation period began October 1, 2010, when the education program began, and ran through December 31, 2011.

\section{Sepsis Bundle Initial Antibiotic Protocol}

The protocol for antimicrobial management of sepsis had 3 steps. The first step was prompt and universal emergent antibiotic therapy for patients with any level of sepsis (sepsis, severe sepsis, septic shock) to be initiated within 1 hour of arrival in the ED or on direct admission. ${ }^{13-16}$ The second step of the antibiotic protocol was composed of additional intravenous antibiotics within 1 to 3 hours, with the specific antiobiotic(s) depending on the potential source(s) of infection. The potential sources were categorized as (a) central nervous system, (b) lung, (c) gastrointestinal, (d) line or skin/bone/joint, and (e) unknown source or urine. Each antimicrobial choice took into consideration the likely causative pathogens (including whether the infection was community or health care acquired), and 
was compatible with Infectious Diseases Society of America guidelines. Direction was given to avoid recently used antibiotics and to seek Infectious Diseases Department consultation for patients with a history of resistant organisms. The third step in the antibiotic protocol included establishing a course of continued antimicrobial treatment, further investigations as indicated, and appropriate source control such as drainage of abscesses. However, it also included ongoing (at least daily) review of available data to facilitate the tailoring of antibiotics, and narrowing of therapy once the causative organism and its antibiotic susceptibilities were identified. Details about the antibiotic selection algorithm before identification of the likely source of sepsis and after identification of the suspected source of sepsis are presented in online Digital Supplemental Content 1 and Content 2, respectively (available at http://ajmq.sagepub.com/supplemental).

\section{Education}

As part of the sepsis initiative, the research team developed a system-wide comprehensive approach to educate all clinicians about sepsis. E-learning sepsis modules were developed incorporating pretesting, clinical vignettes, and posttesting. Sepsis reference pocket guides, sepsis bundle posters, antibiotic algorithm with infusion rates and compatibilities posters, and sepsis reference lists were completed and published on the hospital infection control Web site for ease of access. These references were printed and posted on all clinical care units and in provider work areas. Printed materials are available on request from the corresponding author.

\section{Statistical Analysis}

To evaluate the effectiveness of the initiative, the research team compared sepsis outcomes before the start of the initiative to sepsis outcomes one year after. Five sepsis outcomes were evaluated: (a) sepsis mortality, defined as patients with a sepsis diagnosis who died during hospitalization; (b) the observed to expected (O/E) sepsis mortality ratio; (c) the number of ICU days; (d) hospital length of stay (LOS); and (e) total hospital costs. The O/E ratio was obtained from the University HealthSystem Consortium (UHC), an alliance of academic medical centers and affiliated hospitals. Expected deaths in the ratio are derived from the expected mortality in specific mortality models, which account for patient demographics, admission source, admission status, primary payer, and comorbidities.

The statistical analysis was designed to compare characteristics of patients in the intervention and control periods and to determine whether patients in the intervention period had improved outcomes after controlling for expected mortality and other characteristics. Descriptive analyses presented mean and percent for continuous and categorical variables, respectively, for intervention and control patients. Multivariate analyses of risk of mortality were performed using logistic regression with primary interest in the coefficient for the intervention period indicator. Covariates included sex, race, number of comorbidities, and expected mortality.

Analyses of ICU days, total hospital LOS, and hospital costs were performed using generalized linear models (GLM). Hospital costs were obtained from the hospital's cost accounting database (McKesson, San Francisco, California). This database estimates costs 
using a ratio of costs to charges methodology. Costs, therefore, represent an estimate of the total, fully loaded costs to the hospital. The GLM model was chosen as the statistical model to deal with the heavy skewness in each of the outcomes. The GLM models assumed a gamma family of distributions and a natural log link function. The marginal effect of each variable is reported, which returns the effect to the original (ie, not natural $\log$ ) scale. Again, interest centers on the coefficient for the intervention indicator, and additional covariates included sex, race, number of comorbidities, and expected mortality.

\section{Results}

There were 1331 patients with sepsis during the intervention period and 1401 patients with sepsis during the control period (Table 1). Patients were similar in terms of basic demographics. Intervention patients had slightly more comorbidities than control patients; they also had higher expected mortality than control patients. Still, the overall mortality rate was $23.5 \%$ among patients before the intervention period and $18.9 \%$ among patients during the intervention period. Patients in the intervention period also had a somewhat shorter ICU stay, shorter total hospital LOS, and lower total costs (Table 1). More details about patient comorbidities are presented in Table 2, which suggests that sepsis patients in the intervention period had a higher prevalence of congestive heart failure, peripheral vascular disease, diabetes, and renal disease; the control group had much more cancer.

As seen in Figure 1A, quarterly rates of observed mortality had a falling trend after the intervention began. This, combined with the rising expected mortality rate in the intervention group, led to observable improvements in the quarterly rate of $\mathrm{O} / \mathrm{E}$ mortality (Figure 1B). By the final quarter of the intervention the O/E ratio fell below 1. Average total LOS also fell consistently in each quarter following the intervention (Figure 1C). Average total hospital costs also trended down after the intervention, but did not appear to fall below the control period (Figure 1D).

Multivariate analysis using logistic regression to model the effect of the intervention on mortality suggested that even after controlling for expected mortality, patients in the intervention period had $30 \%$ lower odds of dying (odds ratio $[\mathrm{OR}]=0.70,95 \%$ confidence interval $[\mathrm{CI}]=0.57$ to 0.84 ; Table 3 ). Note that demographics were not significantly associated with risk of mortality, but each additional comorbidity was associated with increased odds of mortality.

Results from multivariate models of ICU days, total LOS, and hospital costs are presented in Table 4. Although demographic variables were not associated with risk of mortality, they had a significant association with resource utilization variables. For example, relative to women, men had a significantly longer average ICU stay $(P=.005)$ and total hospital stay $(P$ $=.001)$. They also incurred more in total costs than women $(P<.0001)$. Increasing numbers of comorbidities were associated with significantly shorter ICU stays, total LOS, and lower costs. This is likely because of the correlation between more comorbidities and mortality. After controlling for demographics, comorbidities, and expected mortality, patients in the intervention period had 1.07 fewer days on average in the ICU ( $95 \% \mathrm{CI}=-1.98$ to -0.16 ) 
and 2.15 fewer hospital days ( $95 \% \mathrm{CI}=-3.45$ to -0.86 ). They also incurred on average $\$ 1949$ less in hospital costs, but this effect was not statistically significant.

\section{Discussion}

The multidisciplinary sepsis quality improvement initiative led to a significant improvement in the study institution's organizational sepsis mortality with an observed sepsis mortality reduction of $4.6 \%$. This translated to a 37\% improvement in the sepsis O/E mortality. After controlling for patient characteristics and expected mortality, patients with sepsis were significantly less likely to die after the intervention $(\mathrm{OR}=0.70)$. Septic patients had a 1.1day shorter ICU duration of stay and a 2.2-day shorter overall hospital stay. Additionally, although not statistically significant, hospital costs were reduced by $\$ 1949$ on average per patient with sepsis. With a baseline mortality rate of $23.5 \%$ and an achieved mortality rate of $18.9 \%$, the research team estimates that 65 lives were saved through the sepsis initiative.

From its inception, the sepsis initiative focused on several integrated dimensions. These consisted of the development of a mandatory online educational program, creation of an institutional algorithm to facilitate the early identification and treatment of sepsis, implementation of an electronic sepsis order set, and the initiation of continuous compliance monitoring through the establishment of an electronic dashboard. Of the basic tenets of the project, education was the primary focus. A mandatory online educational program was developed, as were treatment algorithms and management bundles constructed in accordance with SSC guidelines. ${ }^{8,9}$ These algorithms and bundles were available online and were printed on pocket cards to facilitate wide distribution to faculty, residents, students, and staff. Posters depicting the algorithms and bundles also were created and placed in highly visible areas on each ward or unit.

There is substantial evidence demonstrating the positive impact of sepsis educational programs. In 2008, Ferrer et al described the beneficial effects of an organized sepsis educational program. ${ }^{17}$ As a result of a national educational effort undertaken in Spain to promote bundles of care for severe sepsis and septic shock, both improved guideline compliance and a $4.3 \%$ absolute hospital mortality reduction were realized. ${ }^{17}$ Similarly, Jeon and colleagues reported that an educational program designed to address the management of severe sepsis and septic shock improved both resuscitation bundle compliance and achievement of their corresponding end points. ${ }^{18}$ Furthermore, the creation of an in-hospital sepsis program, which incorporated education of health care personnel and process changes, has been shown to improve guideline adherence and sepsis survival in patients admitted to the ICU. In the pilot study by Girardis and colleagues, in-hospital mortality decreased from $79 \%$ to $32 \%$, largely as a result of the educational efforts of the sepsis program. ${ }^{19}$ With an immediate reduction in mortality after initiation of a formal sepsis educational program, the results of the present study further demonstrate the substantial impact education may possess on improving patient outcomes.

Another factor that contributed to improving patient outcomes was the adoption of an institutional protocol designed to facilitate early recognition and treatment of sepsis. Many similar institutional protocols have yielded positive results. Clinical implementation of a 
comprehensive sepsis treatment protocol has been found to be feasible as well as associated with changes in therapies such as the time to antibiotics, intravenous fluid delivery, and early vasopressor use. ${ }^{20}$ Many reported studies cite the SSC guidelines as the foundation for sepsis protocol development. One such study was reported by Castellanos-Ortega and colleagues in 2010. ${ }^{21}$ Following the implementation of a hospital-wide quality improvement program based on SSC guidelines, the authors reported a sustained and significant decrease in mortality from $57.3 \%$ to $37.5 \%$ in 384 patients in septic shock over a 3-year period. In addition, this patient population also realized a reduction in both hospital (by 4.8 days) and ICU (by 2.6 days) duration of stay. ${ }^{21}$ Kortgen and colleagues also reported a mortality reduction from $53 \%$ to $27 \%{ }^{22}$ through the use of a standard operating procedure or protocol for septic shock. The Generalized Early Sepsis Intervention Strategies Project, a multicenter quality improvement collaborative, demonstrated that patients treated according to a sepsis protocol experienced a decrease in mortality from $42.8 \%$ to $28.8 \%$ and a 5.1 -day decrease in hospital duration of stay. ${ }^{23}$ Furthermore, a large outcomes survey of early sepsis management after implementation of sepsis protocols at 12 centers including 1298 patients showed a substantial mortality reduction of $20.3 \%$. Sepsis mortality decreased from $44.8 \%$ before to $24.5 \%$ after implementation of sepsis programs. An additional key finding in this study was the fact that the rapid institution of interventions uniformly improved patient outcomes. ${ }^{7}$ These results are consistent with the initial groundbreaking results that Rivers et al reported in 2001, which noted improved mortality from $46.5 \%$ to $30.5 \%$ after implementation of EGDT. ${ }^{6}$ Although in the present study the absolute mortality reduction was only $4.6 \%$, the $23.5 \%$ mortality in control patients was considerably less than most previously reported studies. It is not precisely clear why the study institution's baseline sepsis mortality compared favorably to other reports. In fact, internal data that suggested a high rate of undiagnosed sepsis prompted the research team's efforts to seek ways to improve sepsis mortality. It is possible that the emphasis placed on early detection, albeit informal, led to more aggressive early intervention and improved mortality. Regardless, the improvement to an overall sepsis mortality of $18.9 \%$ compares favorably with the outcomes of other published reports.

Instrumental in the development of sepsis protocols is the adoption of a sepsis resuscitation bundle. This requires close collaboration between emergency medicine and critical care providers, as well as collaboration with pharmacists to facilitate the prompt availability of anchor antibiotics at the bedside. This collaboration has been found to reliably facilitate the achievement of EGDT end points in real-world sepsis resuscitation. ${ }^{24}$ The implementation of a sepsis resuscitation protocol has been demonstrated to significantly reduce the time until therapeutic interventions occur in daily practice. ${ }^{22}$ It is this time to intervention that has garnered a great deal of attention in recent years. Noncompliance with a 6-hour sepsis bundle has been associated with a greater than 2-fold increase in hospital mortality, and noncompliance at 24 hours has been associated with a $76 \%$ increase in hospital mortality. ${ }^{25}$ On the contrary, in 498 patients with severe sepsis or septic shock, compliance with a sepsis resuscitation bundle at 18 hours demonstrated a significant mortality benefit with mortality decreased from $47.3 \%$ to $37.1 \%{ }^{26}$

Another early intervention that contributed to the improved outcomes realized in the present study was the creation of electronic sepsis, severe sepsis, and septic shock order sets. Other 
studies also have found sepsis order sets to be beneficial. In one study, implementation of a standardized order set for the management of septic shock in the ED has been associated with statistically more rigorous fluid resuscitation, greater administration of appropriate initial antibiotics, decreased 28-day mortality from $48.3 \%$ to $30.0 \%$, and decreased hospital duration of stay from 12.1 to 8.9 days. ${ }^{27}$ Similarly, the implementation of a hospital-wide standardized order set for the management of bacteremic severe sepsis has been associated with greater fluid administration, improved antibiotic therapy, decreased incidence of organ failure, improved in-hospital mortality from $55.0 \%$ to $39.5 \%$, and decreased duration of hospital stay from 28.7 to 22.4 days. ${ }^{28}$ Undoubtedly, the electronic order set facilitates appropriate initiation of sepsis resuscitation bundles as well as ensures adherence to the sepsis protocol.

This study has several limitations. First, the research team identified sepsis patients both retrospectively and prospectively based on review of documented primary and secondary diagnosis codes. Patients with sepsis, severe sepsis, and/or septic shock were not included in the study if clinician documentation was inadequate or inaccurate. For example, patients with documented bacteremia would not be captured if documentation was annotated as bacteremia rather than sepsis related to bacteremia. Deficiencies in documentation were recognized early and the educational program was specifically designed to improve documentation. Nonetheless, it remains possible that some patients with the varying stages of sepsis were omitted because of inadequate documentation. Another limitation is that the focus included all patients with sepsis. As such, the research team did not stratify patients with severe sepsis and septic shock. Also, the study institution was concurrently participating in other quality initiatives to improve the recognition and management of sepsis, including a UHC collaborative and a randomized trial of severe sepsis and septic shock patients presenting to the ED (NIGMSP50GMO76659). ${ }^{29}$ Thus, some of the improvement in outcomes may have been attributable to other interventions.

\section{Conclusions}

The establishment of an institutional multidisciplinary sepsis quality improvement initiative resulted in a reduction in overall in-hospital sepsis mortality to less than $20 \%$ and a $37 \%$ improvement in $\mathrm{O} / \mathrm{E}$ sepsis mortality. Through a multifaceted approach including mandatory education, protocol development, the identification of standard anchor antibiotics, the creation of electronic order sets, and continuous compliance monitoring, sepsis patients in the intervention period had a $30 \%$ lower risk of dying. Both ICU days and overall hospital LOS were significantly decreased. Although estimated direct costs were reduced, this effect was not statistically significant. As with any successful intervention, the challenge will be to sustain these improvements.

\section{Supplementary Material}

Refer to Web version on PubMed Central for supplementary material. 


\section{Acknowledgments}

This work benefited from the contributions of Ann N. Peric, MPA, and Erin Sarsfield, MSN, RN, who provided valuable input through programming, data collection, report writing, and project management.

\section{Funding}

The authors received no financial support for the research, authorship, and/or publication of this article.

\section{References}

1. Angus DC, Wax RS. Epidemiology of sepsis: an update. Crit Care Med. 2001; 29(7 suppl):S109_ S116. [PubMed: 11445744]

2. Bone RC, Balk RA, Cerra FB, et al. Definitions for sepsis and organ failure and guidelines for the use of innovative therapies in sepsis. The ACCP/SCCM Consensus Conference Committee. American College of Chest Physicians/Society of Critical Care Medicine. Chest. 1992; 101:16441655. [PubMed: 1303622]

3. Friedman G, Silva E, Vincent JL. Has the mortality of septic shock changed with time. Crit Care Med. 1998; 26:2078-2086. [PubMed: 9875924]

4. Alberti C, Brun-Buisson C, Burchardi H, et al. Epidemiology of sepsis and infection in ICU patients from an international multicentre cohort study. Intensive Care Med. 2002; 28:108-121. [PubMed: 11907653]

5. Martin GS, Mannino DM, Eaton S, Moss M. The epidemiology of sepsis in the United States from 1979 through 2000. N Engl J Med. 2003; 348:1546-1554. [PubMed: 12700374]

6. Rivers E, Nguyen B, Havstad S, et al. Early goal-directed therapy in the treatment of severe sepsis and septic shock. N Engl J Med. 2001; 345:1368-1377. [PubMed: 11794169]

7. Otero RM, Nguyen HB, Huang DT, et al. Early goal-directed therapy in severe sepsis and septic shock revisited: concepts, controversies, and contemporary findings. Chest. 2006; 130:1579-1595. [PubMed: 17099041]

8. Dellinger RP, Carlet JM, Masur H, et al. Surviving Sepsis Campaign guidelines for management of severe sepsis and septic shock. Crit Care Med. 2004; 32:858-873. [PubMed: 15090974]

9. Dellinger RP, Levy MM, Carlet JM, et al. Surviving Sepsis Campaign: international guidelines for management of severe sepsis and septic shock: 2008. Intensive Care Med. 2008; 34:17-60. [PubMed: 18058085]

10. Levy MM, Dellinger RP, Townsend SR, et al. The Surviving Sepsis Campaign: results of an international guideline-based performance improvement program targeting severe sepsis. Intensive Care Med. 2010; 36:222-231. [PubMed: 20069275]

11. Zhao H, Heard SO, Mullen MT, et al. An evaluation of the diagnostic accuracy of the 1991 American College of Chest Physicians/Society of Critical Care Medicine and the 2001 Society of Critical Care Medicine/European Society of Intensive Care Medicine/American College of Chest Physicians/American Thoracic Society/Surgical Infection Society sepsis definition. Crit Care Med. 2012; 40:1700-1706. [PubMed: 22610176]

12. Poeze M, Ramsay G, Gerlach H, Rubulotta F, Levy M. An international sepsis survey: a study of doctors' knowledge and perception about sepsis. Crit Care. 2004; 8:R409-R413. [PubMed: 15566585]

13. Harbarth S, Garbino J, Pugin J, Romand JA, Lew D, Pittet D. Inappropriate initial antimicrobial therapy and its effect on survival in a clinical trial of immunomodulating therapy for severe sepsis. Am J Med. 2003; 115:529-535. [PubMed: 14599631]

14. Ibrahim EH, Sherman G, Ward S, Fraser VJ, Kollef MH. The influence of inadequate antimicrobial treatment of bloodstream infections on patient outcomes in the ICU setting. Chest. 2000; 118:146155. [PubMed: 10893372]

15. Leibovici L, Shraga I, Drucker M, Konigsberger H, Samra Z, Pitlik SD. The benefit of appropriate empirical antibiotic treatment in patients with bloodstream infection. J Intern Med. 1998; 244:379386. [PubMed: 9845853] 
16. Kollef MH, Sherman G, Ward S, Fraser VJ. Inadequate antimicrobial treatment of infections: a risk factor for hospital mortality among critically ill patients. Chest. 1999; 115:462-474. [PubMed: 10027448]

17. Ferrer R, Artigas A, Levy MM, et al. Improvement in process of care and outcome after a multicenter severe sepsis educational program in Spain. JAMA. 2008; 299:2294-2303. [PubMed: 18492971]

18. Jeon K, Shin TG, Sim MS, et al. Improvements in compliance with resuscitation bundles and achievement of end points after an educational program on the management of severe sepsis and septic shock. Shock. 2012; 37:463-467. [PubMed: 22301605]

19. Girardis M, Rinaldi L, Donno L, et al. Effects on management and outcome of severe sepsis and septic shock patients admitted to the intensive care unit after implementation of a sepsis program: a pilot study. Crit Care. 2009; 13:R143. [PubMed: 19728879]

20. Shapiro NI, Howell MD, Talmor D, et al. Implementation and outcomes of the Multiple Urgent Sepsis Therapies (MUST) protocol. Crit Care Med. 2006; 34:1025-1032. [PubMed: 16484890]

21. Castellanos-Ortega A, Suberviola B, Garcia-Astudillo LA, et al. Impact of the Surviving Sepsis Campaign protocols on hospital length of stay and mortality in septic shock patients: results of a three-year follow-up quasi-experimental study. Crit Care Med. 2010; 38:1036-1043. [PubMed: 20154597]

22. Kortgen A, Niederprum P, Bauer M. Implementation of an evidence-based "standard operating procedure" and outcome in septic shock. Crit Care Med. 2006; 34:943-949. [PubMed: 16484902]

23. Cannon CM, Holthaus CV, Zubrow MT, et al. The GENESIS Project (GENeralized Early Sepsis Intervention Strategies): a multicenter quality improvement collaborative. J Intensive Care Med. 2013; 28:355-368. [PubMed: 22902347]

24. Trzeciak S, Dellinger RP, Abate NL, et al. Translating research to clinical practice: a 1-year experience with implementing early goal-directed therapy for septic shock in the emergency department. Chest. 2006; 129:225-232. [PubMed: 16478835]

25. Gao F, Melody T, Daniels DF, Giles S, Fox S. The impact of compliance with 6-hour and 24-hour sepsis bundles on hospital mortality in patients with severe sepsis: a prospective observational study. Crit Care. 2005; 9:R764-R770. [PubMed: 16356225]

26. Coba V, Whitmill M, Mooney R, et al. Resuscitation bundle compliance in severe sepsis and septic shock: improves survival, is better late than never. J Intensive Care Med. 2011; 26:304-343. [PubMed: 21220270]

27. Micek ST, Roubinian N, Heuring T, et al. Before-after study of a standardized hospital order set for the management of septic shock. Crit Care Med. 2006; 34:2707-2713. [PubMed: 16943733]

28. Thiel SW, Asghar MF, Micek ST, Reichley RM, Doherty JA, Kollef MH. Hospital-wide impact of a standardized order set for the management of bacteremic severe sepsis. Crit Care Med. 2009; 37:819-824. [PubMed: 19237883]

29. The ProCESS Investigators. Yealy DM, Kellum JA, Huang DT, et al. A randomized trial of protocol-based care for early septic shock. N Engl J Med. 2014; 370:1683-1693. [PubMed: 24635773] 

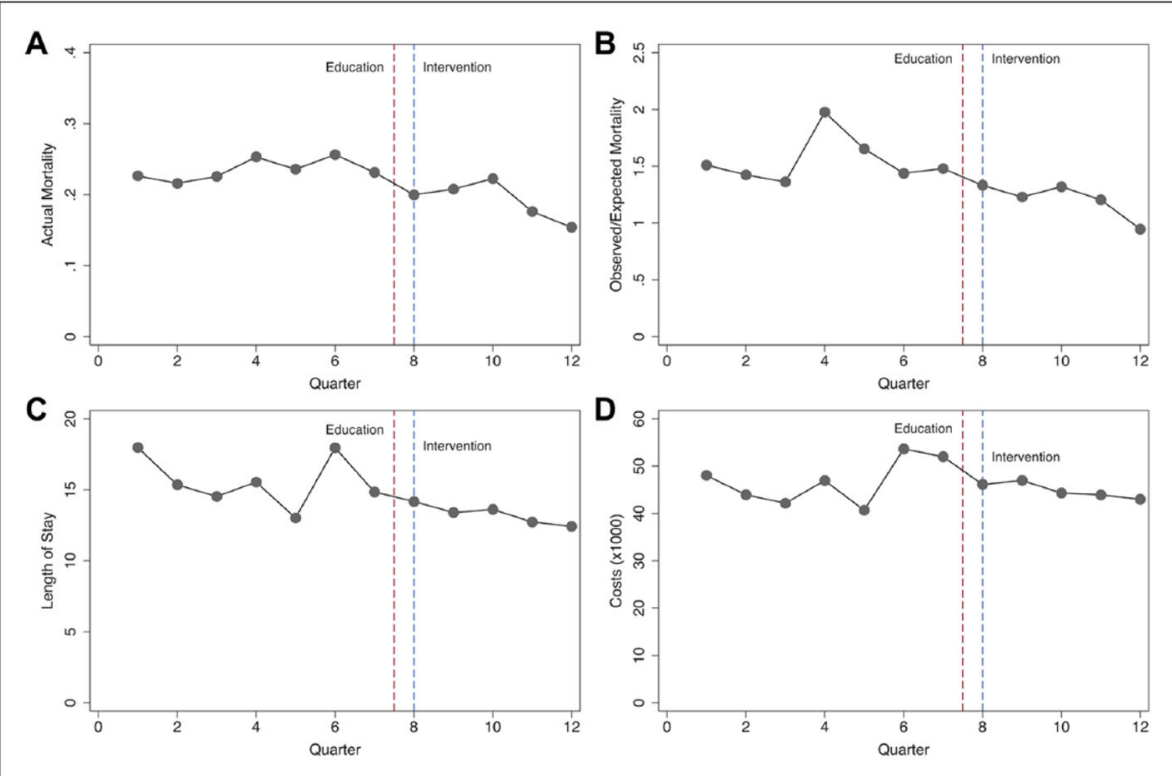

Figure 1.

Quarterly outcomes after sepsis intervention.

(A) Trend in observed mortality rate for patients with sepsis by quarter, 2009 to 2011. (B) Trend in ratio of observed mortality to expected mortality by quarter, 2009 to 2011 . (C) Trend in average length of hospital stay for patients with sepsis by quarter, 2009 to 2011. (D) Trend in average hospital costs for patients with sepsis by quarter, 2009 to 2011. 
Table 1

Characteristics of Sepsis Patients, Stratified by Intervention Period. ${ }^{a}$

\begin{tabular}{lcccc}
\hline Variable & Control (N = 1401) & Intervention (N= 1331) & All (N = 2732) & $\boldsymbol{P}$ Value \\
\hline Sex & & & & .895 \\
$\quad$ Male & $57.6 \%$ & $57.9 \%$ & $57.7 \%$ & \\
Female & $42.4 \%$ & $42.1 \%$ & $42.3 \%$ & \\
Race & & & & .208 \\
$\quad$ White & $88.3 \%$ & $86.7 \%$ & $87.5 \%$ & \\
Nonwhite & $11.7 \%$ & $13.3 \%$ & $12.5 \%$ & \\
Comorbidities & & & & .608 \\
0 & $20.0 \%$ & $20.4 \%$ & $20.2 \%$ & \\
1 & $40.5 \%$ & $38.2 \%$ & $39.4 \%$ & \\
2 & $26.6 \%$ & $27.3 \%$ & $27.0 \%$ & \\
$\geq 3$ & $12.8 \%$ & $14.0 \%$ & $13.4 \%$ & \\
Mortality risk & & & & .001 \\
$0 \%$ to $5 \%$ & $35.8 \%$ & $28.9 \%$ & $32.4 \%$ & \\
$5.1 \%$ to $10 \%$ & $32.4 \%$ & $36.7 \%$ & $34.5 \%$ & \\
$>10 \%$ & $31.8 \%$ & $34.4 \%$ & $33.1 \%$ & \\
Outcomes & & & & .0351 \\
Mortality & $23.5 \%$ & $18.9 \%$ & $21.2 \%$ & .003 \\
LOS (days) & 15.6 & 13.1 & 14.4 & .0015 \\
ICU days & 6.6 & 5.5 & 6.1 & .4532 \\
Costs & $\$ 463631$ & $\$ 443630$ & &
\end{tabular}

Abbreviations: ICU, intensive care unit; LOS, length of stay.

${ }^{a}$ The control period was January 1, 2009, to September 30, 2010. The intervention period was October 1, 2010, to December 31, 2011. 
Table 2

Comorbidities Among Sepsis Patients Stratified by Intervention Period. ${ }^{a}$

\begin{tabular}{lrrrc}
\hline Comorbidity & Control (N = 1401) & Intervention $(\mathbf{N}=\mathbf{1 3 3 1})$ & All (N = 2732) & $\boldsymbol{P}$ Value \\
\hline Acute myocardial infarction & $13.0 \%$ & $12.5 \%$ & $12.8 \%$ & .7285 \\
Congestive heart failure & $13.8 \%$ & $15.3 \%$ & $14.6 \%$ & .2734 \\
Peripheral vascular disease & $3.9 \%$ & $5.6 \%$ & $4.7 \%$ & .0351 \\
Cerebrovascular disease & $6.9 \%$ & $7.1 \%$ & $7.0 \%$ & .8271 \\
Dementia & $0.1 \%$ & $0.2 \%$ & $0.2 \%$ & .6136 \\
Chronic obstructive pulmonary disease & $13.0 \%$ & $14.4 \%$ & $13.7 \%$ & .3011 \\
Rheumatoid disease & $2.9 \%$ & $2.0 \%$ & $2.5 \%$ & .1003 \\
Peptic ulcer disease & $1.7 \%$ & $1.7 \%$ & $1.7 \%$ & .976 \\
Mild liver disease & $1.0 \%$ & $1.1 \%$ & $1.1 \%$ & .7449 \\
Diabetes & $17.8 \%$ & $20.1 \%$ & $19.0 \%$ & .1269 \\
Diabetes + complications & $4.6 \%$ & $6.0 \%$ & $5.3 \%$ & .1102 \\
Hemiplegia/paraplegia & $3.3 \%$ & $3.2 \%$ & $3.3 \%$ & .9382 \\
Renal disease & $11.2 \%$ & $14.4 \%$ & $12.7 \%$ & .0137 \\
Cancer & $30.0 \%$ & $23.0 \%$ & $26.6 \%$ & $<.0001$ \\
Moderate/severe liver disease & $4.7 \%$ & $4.6 \%$ & $4.6 \%$ & .874 \\
Metastatic cancer & $7.9 \%$ & $7.8 \%$ & $7.9 \%$ & .9156 \\
Acquired immune deficiency syndrome & $0.0 \%$ & $0.0 \%$ & $0.0 \%$ & - \\
\hline
\end{tabular}




Table 3

Determinants of In-Hospital Mortality Among Patients With Sepsis. ${ }^{a}$

\begin{tabular}{|c|c|c|c|c|}
\hline \multirow[b]{2}{*}{ Variable } & \multirow[b]{2}{*}{ Odds Ratio } & \multicolumn{2}{|c|}{ 95\% Confidence Interval } & \multirow[b]{2}{*}{$P$ Value } \\
\hline & & Lower & Upper & \\
\hline \multicolumn{5}{|l|}{ Sex } \\
\hline Female & Reference & & & \\
\hline Male & 1.21 & 0.99 & 1.47 & .06 \\
\hline \multicolumn{5}{|l|}{ Race } \\
\hline White & Reference & & & \\
\hline Nonwhite & 1.12 & 0.84 & 1.49 & .457 \\
\hline \multicolumn{5}{|l|}{ Comorbidities } \\
\hline 0 & Reference & & & \\
\hline 1 & 1.82 & 1.36 & 2.44 & $<.0001$ \\
\hline 2 & 1.88 & 1.38 & 2.55 & $<.0001$ \\
\hline$\geq 3$ & 1.91 & 1.34 & 2.71 & $<.0001$ \\
\hline \multicolumn{5}{|l|}{ Mortality risk } \\
\hline $0 \%$ to $5 \%$ & Reference & & & \\
\hline $5.1 \%$ to $10 \%$ & 1.72 & 1.31 & 2.27 & $<.0001$ \\
\hline$>10 \%$ & 4.84 & 3.74 & 6.25 & $<.0001$ \\
\hline Intervention & 0.70 & 0.57 & 0.84 & $<.0001$ \\
\hline
\end{tabular}

Intervention indicates the intervention period from October 1, 2010, to December 31, 2011. 


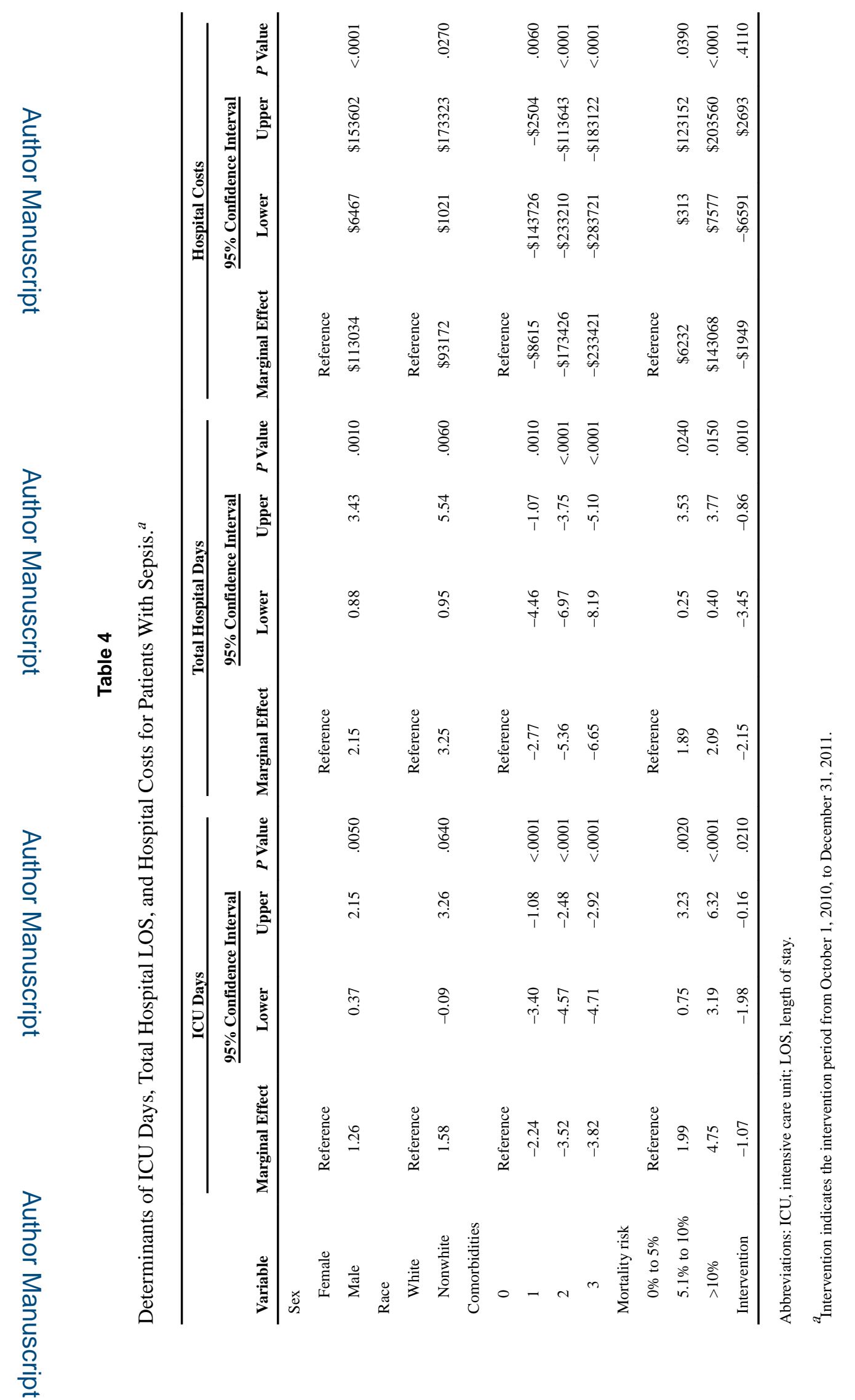

Am J Med Qual. Author manuscript; available in PMC 2017 December 15. 